\title{
Communicable Diseases Report, NSW, May and June 2010
}

\author{
Communicable Diseases Branch \\ NSW Department of Health
}

For updated information, including data and facts on specific diseases, visit www.health.nsw.gov.au and click on Public Health and then Infectious Diseases. The communicable diseases site is available at: http://www.health.nsw.gov.au/publichealth/ infectious/index.asp.

Figure 3 and Tables 1 and 2 show reports of communicable diseases received through to the end of May and June 2010 in New South Wales (NSW).

\section{Enteric infections \\ Typhoid}

Three cases of typhoid fever were reported in NSW in May and June 2010. All cases reported a history of overseas travel to India during their exposure period. Eighteen cases have been reported in NSW so far this year. For the same period in 2009, 22 cases were reported.

Typhoid is caused by the bacteria Salmonella Typhi and is transmitted by the faecal-oral route, primarily by ingesting food or water contaminated by faeces or urine. The symptoms of typhoid fever may include fever, headache, general discomfort and lack of appetite; a dry cough and constipation or diarrhoea may also occur. Typhoid infections in NSW are usually acquired while people are travelling overseas to countries where they may ingest contaminated water or food.

\section{Outbreaks of foodborne disease}

Seven outbreaks of suspected foodborne disease were reported in May and June 2010. Data from public health investigations are available on four of these outbreaks which affected 35 people. Salmonella was the cause of three of the four outbreaks which were associated with foods including chicken curry, chicken kebab, hummus, tabouli, and chicken in cheese sauce. Campylobacter jejuni was the cause of the fourth outbreak, suspected to be associated with undercooked chicken.

\section{Outbreaks of gastroenteritis in institutional settings}

Eighty-four outbreaks of gastroenteritis in institutions were reported in May and June 2010, affecting 1275 people. Of these, 40 outbreaks occurred in aged-care facilities, 30 in child-care centres, nine in hospitals, two in schools, two in residential facilities and one at a naval base. Eighty-three outbreaks appear to have been caused by person-to-person spread of a viral illness and one was foodborne.

Viral gastroenteritis tends to peak in winter months, with up to 15 outbreaks per week reported in peak months.

\section{Gastroenteritis in the community}

The number of patients presenting with gastrointestinal illness to emergency departments in NSW increased slightly but remains within the usual range for this time of year (includes data from 56 NSW emergency departments) (Figure 1).

\section{Respiratory infections \\ Legionnaires' disease}

Seven cases of Legionnaires' disease caused by L. pneumophila were reported in NSW in May and June 2010. No common links between cases were identified through local public health unit investigations. Four cases occurred in residents of metropolitan Sydney and three in residents of regional NSW. To date this year, 40 cases of Legionnaires' disease (18 caused by L. pneumophila) have been reported in NSW. For the same period in 2009, 20 legionellosis cases (seven caused by L. pneumophila) were reported. For the same period in 2009,52 cases were reported.

\section{Influenza}

The number of patients presenting with influenza-like illness in NSW remained low (includes information from 56 NSW emergency departments) (Figure 2).

\section{Seasonal influenza vaccination for young children}

The Australian Chief Medical Officer, Professor Jim Bishop, has advised that children aged from 6 months to less than 5 years of age can now be vaccinated against influenza using Vaxigrip ${ }^{\circledR}$ or Influvac ${ }^{\circledR}$ seasonal influenza vaccine. Seasonal influenza vaccination in this age group had been halted following reports of an increase in the rate of febrile convulsions in the 24 hours after vaccination with 2010 seasonal influenza vaccines. 


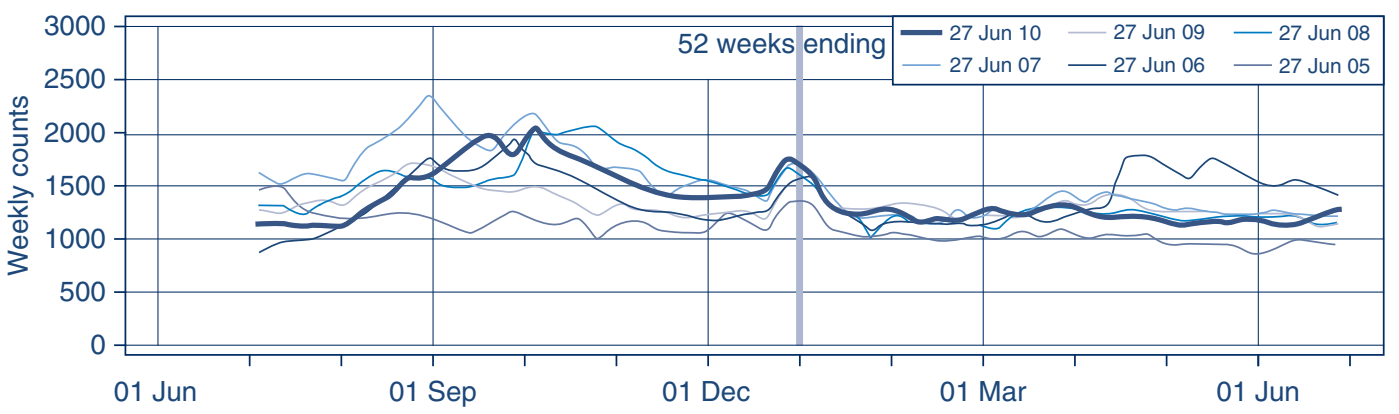

Figure 1. Total weekly counts of emergency department visits for gastrointestinal illness, for the 12 months to 27 June 2010 (thick line), compared with each of the 5 previous years (coloured lines) (includes data from 56 NSW emergency departments).

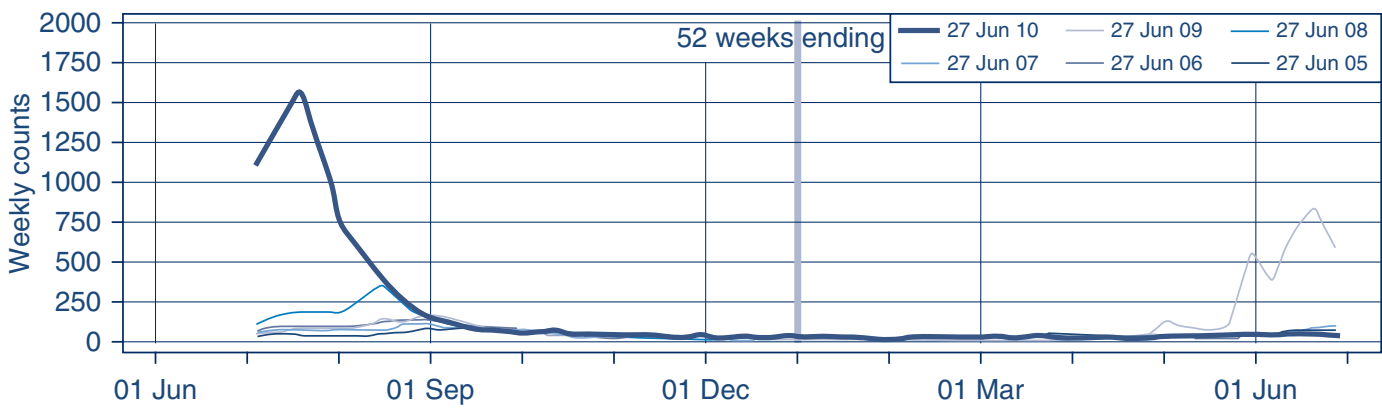

Figure 2. Total weekly counts of emergency department visits for influenza-like illness, for the 12 months to 27 June 2010 (thick line), compared with each of the 5 previous years (coloured lines) (includes data from 56 NSW emergency departments).

Epidemiological investigations have confirmed that the increase in rates of febrile convulsions has only been documented for the 2010 Fluvax ${ }^{\circledR}$ and Fluvax ${ }^{\circledR}$ Junior seasonal influenza vaccines. Investigations from New Zealand (where Vaxigrip ${ }^{\circledR}$ has been widely used during the 2010 influenza season) did not demonstrate any increase in rates of febrile convulsions; the analysis of Australian data for Influvac ${ }^{\circledR}$ also does not demonstrate any increase in rates of febrile convulsions.

As a result of these investigations it is recommended that children aged from 6 months to less than 5 years of age may be vaccinated with Vaxigrip ${ }^{\circledR}$ or Influvac ${ }^{\circledR}$ following a discussion of the risks and benefits of these vaccines with parents and guardians. This includes both children at risk of medical complications of influenza and healthy children. Vaccination of children in this age group with Fluvax ${ }^{\circledR}$ and Fluvax ${ }^{\circledR}$ Junior is not recommended due to the identified increase risk of febrile convulsions.

Further information can be found on the Australian Department of Health website: http:/www.health.gov.au/

\section{Vaccine-preventable diseases Measles}

Two cases of measles were reported in adults who had recently returned from overseas travel to Italy and Sri Lanka. One case was unimmunised and the other had an unknown vaccination history. There have been no reports of secondary transmission associated with these cases.

Eight cases of measles have been reported in NSW this year (seven of these had travelled overseas and one was a contact of a known case). In 2009, nine cases were reported in NSW for the corresponding period.

As previously reported, most cases of measles in NSW are seen in travellers who return with the infection from countries where measles is endemic and who are exposed to a known case. Many people who were born since 1966 and before the mid-80s are not immune to measles because they have neither acquired the measles infection nor received two doses of a measles vaccine. Measles vaccine is now routinely given to infants at 12 months and at 4 years, and this confers long-lasting immunity.

\section{Meningococcal disease}

Eight cases of meningococcal disease were reported in NSW in May and June 2010. The ages of the affected people ranged from 0 to 82 years (five were aged less than 5 years, one was aged $25-30$ years and two were aged over 50 years). Five cases were female. Of the eight cases, four were due to serogroup B, one (an elderly woman with unknown vaccination status) was due to serogroup $C$, and for three clinically diagnosed cases the serogroup was unknown. For the same period in 2009, 16 cases were 
reported. Twenty-nine cases and three deaths due to meningococcal disease have been reported so far in 2010. Fortytwo cases were reported between January and June 2009.

A free vaccine is available for infants at 12 months of age. Consequently, serogroup $\mathrm{C}$ meningococcal disease is now mainly seen in adults and in unimmunised children. In NSW in $2009,80 \%$ of cases of meningococcal disease (where serogroup was known) were caused by serogroup B, for which there is no vaccine.

\section{Haemophilus influenza type $b$ invasive infection}

Two cases of Haemophilus influenza type b invasive infection (Hib) were reported in NSW in May and June 2010 (one an infant and one a child aged 3 years). Both cases were fully vaccinated for age; one case resided in regional NSW and the other in metropolitan Sydney. No other cases have been reported this year in NSW.

For the same period in 2009, two cases of Hib were reported in children aged less than 5 years and four cases in children aged over 5 years.

Since the introduction of a vaccine in 1993, invasive Hib infection has become rare. Hib disease is caused by infection with Haemophilus influenzae type b bacteria. Infection can cause meningitis, epiglottitis (severe swelling of the epiglottis at the back of the throat) and pneumonia. These conditions can develop quickly, and meningitis and epiglottitis can be fatal.

\section{Sexually transmissible infections}

Lymphogranuloma venereum

Six cases of lymphogranuloma venereum were reported in metropolitan Sydney in May and June 2010. The infections were acquired locally and all cases were men aged between 32 and 52 years. Sixteen cases have been reported to date in 2010. In 2009, three cases were reported.

Lymphogranuloma venereum is a rare sexually transmitted chlamydial infection that spreads through unprotected vaginal, anal or oral sexual contact, especially if there is trauma to the skin or mucous membranes. Men who have sex with men, especially those who have unprotected anal sex, are at greatest risk. The bacteria that cause lymphogranuloma venereum are rare types of chlamydia, however lymphogranuloma venereum infection is a more aggressive disease than common chlamydia infections. The infection is treated with an extended course of antibiotics. 
Figure 3. Reports of selected communicable diseases, NSW, January 2004 to June 2010, by month of onset. Preliminary data: case counts in recent months may increase because of reporting delays.

Laboratory-confirmed cases only, except for measles, meningococcal disease and pertussis.

BFV, Barmah Forest virus infection; RRV, Ross River virus infections; lab conf, laboratory confirmed;

Men $\mathrm{Gp} C$ and $\mathrm{Gp} B$, meningococcal disease due to serogroup $C$ and serogroup $B$ infection; other/unk, other or unknown serogroups.

NB: Multiple series in graphs are stacked, except gastroenteritis outbreaks.

NB: Outbreaks are more likely to be reported by nursing homes \& hospitals than by other institutions.

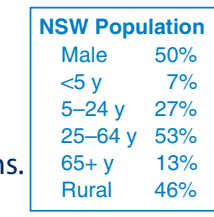

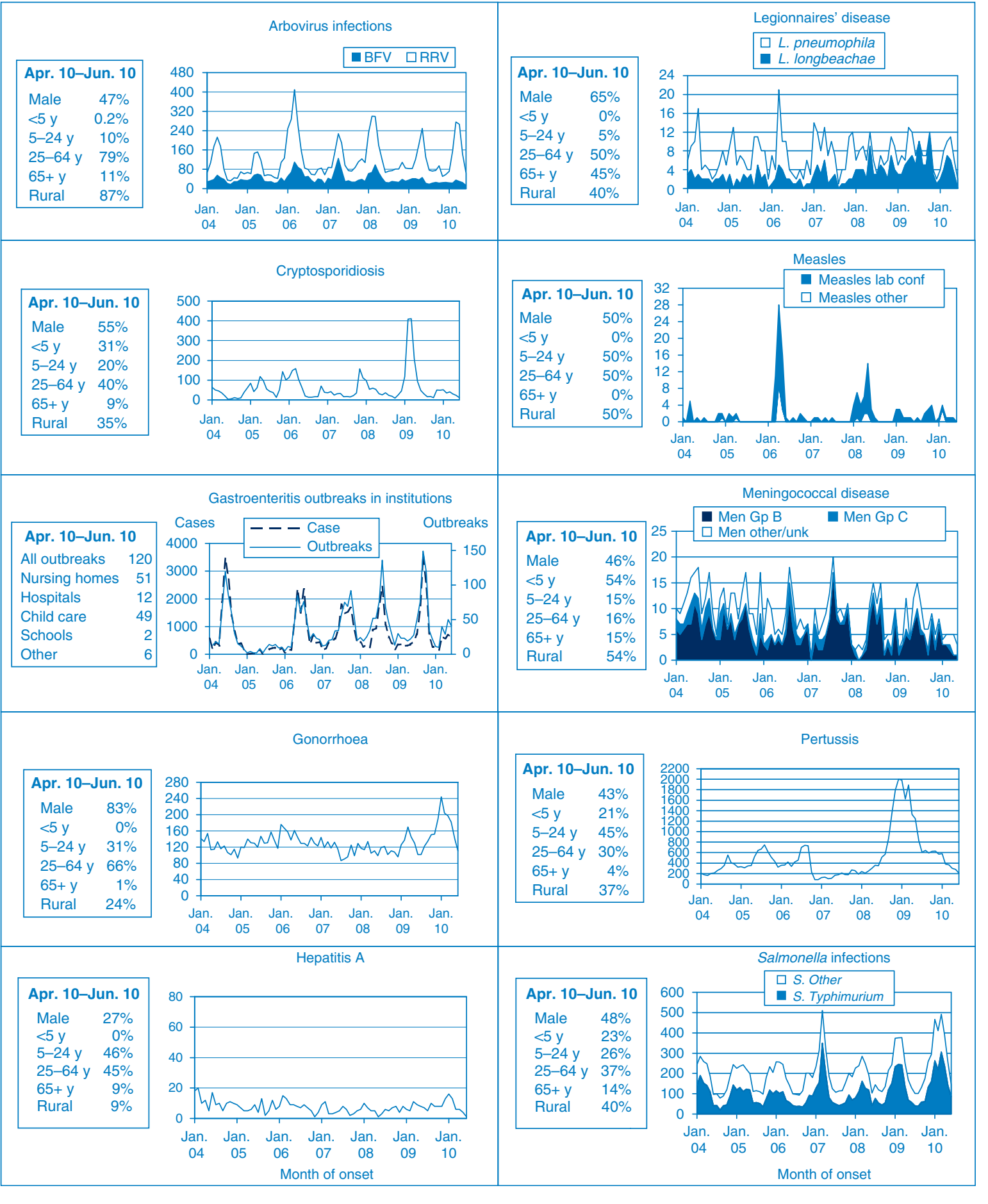




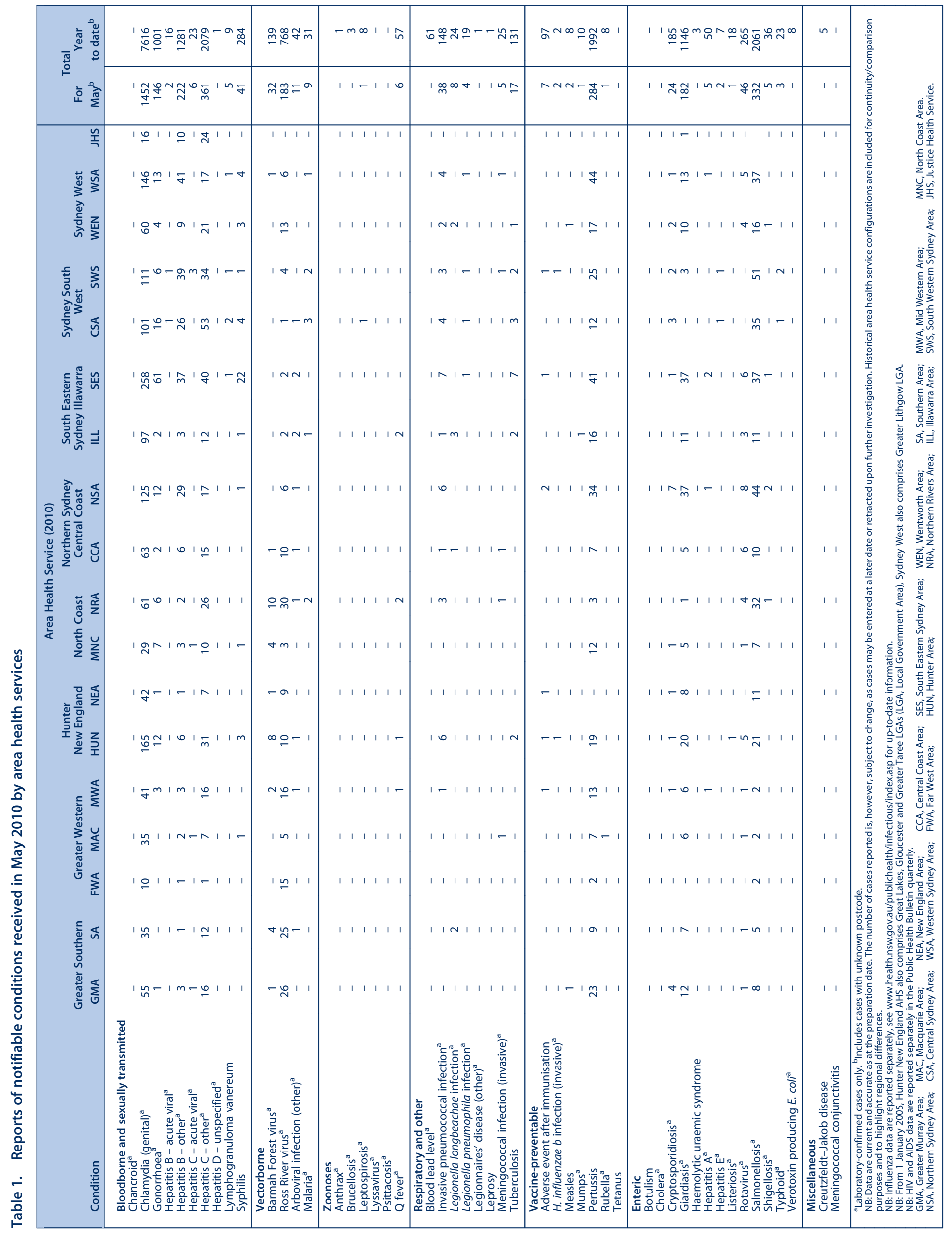




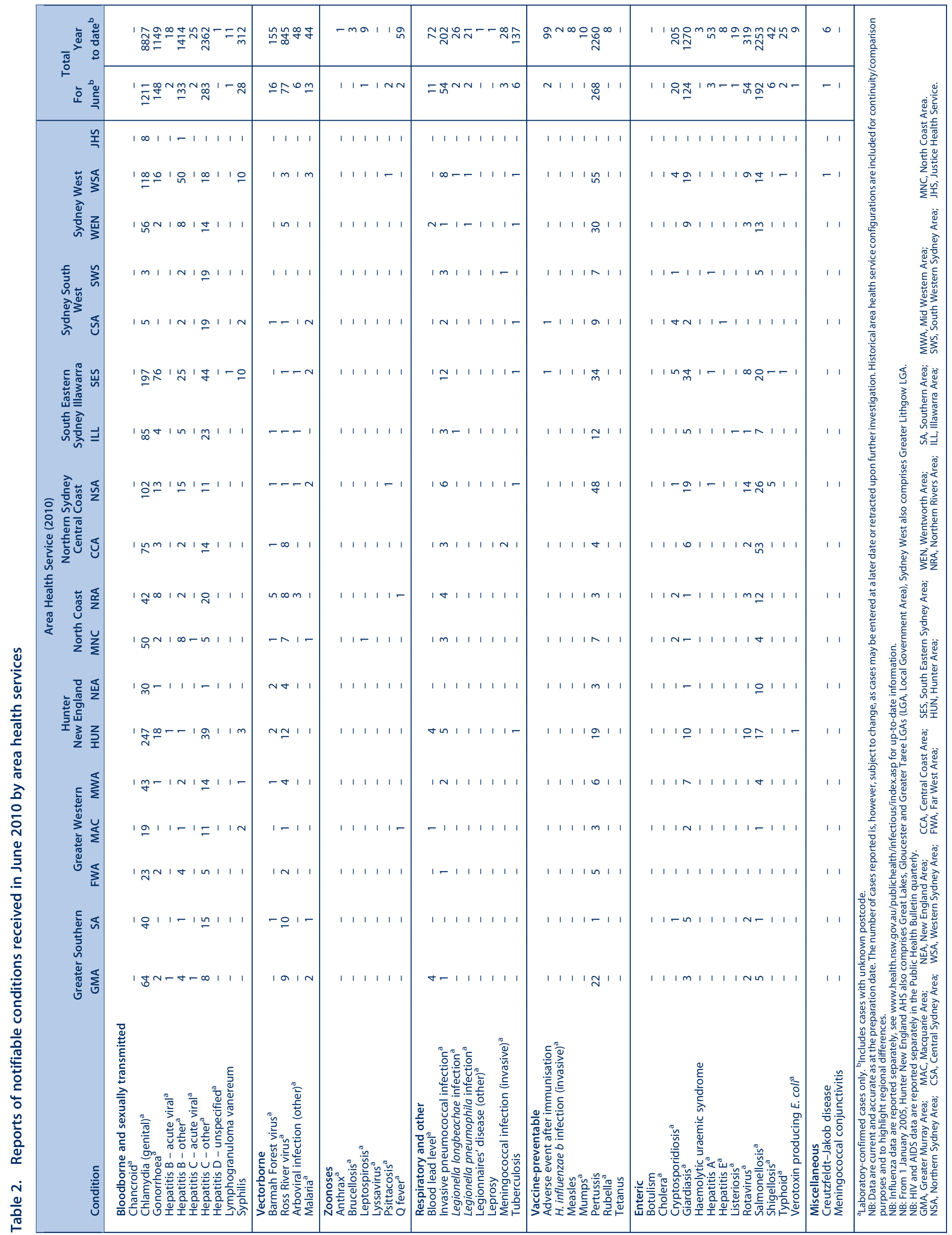

